\title{
Specimen Preparation for Scanning Electron Microscope Using a Converted Sample Stage
}

\author{
*Correspondence to: \\ Bae TS, \\ Tel: +82-63-711-4515 \\ Fax: +82-63-270-4308 \\ E-mail: chemipia@kbsi.re.kr \\ Received October 31, 2015 \\ Revised November 30, 2015 \\ Accepted November 30, 2015
}

\author{
Hyelan Kim, Hyo-Sik Kim ${ }^{1}$, Seungmin Yu, Tae-Sung Bae* \\ Korea Basic Science Institute, Jeonju Center, Jeonju 54653, Korea \\ ${ }^{1}$ Korea Basic Science Institute, Instrumentation Development Support Group, Daejeon 34133, Korea
}

\begin{abstract}
This study introduces metal coating as an effective sample preparation method to remove charge-up caused by the shadow effect during field emission scanning electron microscope (FE-SEM) analysis of dynamic structured samples. During a FE-SEM analysis, charge-up occurs when the primary electrons (input electrons) that scan the specimens are not equal to the output electrons (secondary electrons, backscattered electrons, auger electrons, etc.) generated from the specimens. To remove charge-up, a metal layer of $\mathrm{Pt}, \mathrm{Au}$ or $\mathrm{Pd}$ is applied on the surface of the sample. However, in some cases, charge-up still occurs due to the shadow effect. This study developed a coating method that effectively removes chargeup. By creating a converted sample stage capable of simultaneous tilt and rotation, the shadow effect was successfully removed, and image data without charge-up were obtained.
\end{abstract}

Key Words: Shadow effect, Charge-up, Converted sample stage, Field emission scanning electron microscope

\section{INTRODUCTION}

Field emission scanning electron microscope (FE-SEM) is used in various fields of research because of its superior resolution to optical microscopy, simple preparation of samples, support for analysis of various sample image sizes, and fast data acquisition. The development of FE-SEM equipment has enabled high-quality image analysis, but fundamental problems such as charge-up (Shaffner \& Van Veld, 1990; Thong et al., 2001; Kim et al., 2010), thermal damage (Egerton et al., 2004), and contamination (Hirsh et al., 1994; Postek, 1996; Vladár \& Postek, 2005) must be resolved for a high-quality SEM image analysis. Charge-up is the most common among these problems and the greatest obstruction in image analysis. This occurs when the number of electrons landing on the sample is not equal to the number of electrons escaping from the sample. To remove chargeup, the sample surface is coated with a thin layer $(\sim 10 \mathrm{~nm})$ of metal materials ( $\mathrm{Pt}, \mathrm{Au}, \mathrm{Os}, \mathrm{C}$, etc.). The uniform metal coating layer on the sample surface serves as an electrical conductive path connecting the sample and the stub surface, thereby effectively removing charge-up. However, for dynamic structured samples having a small contact area with the stub surface, the shadow effect (Clay \& Peace, 2011) occurs because the surface is not fully covered by the coating layer. As such, charge-up is not effectively removed when a metal coating is applied to dynamic structured samples since the resulting coating layer is non-uniform.

The ion sputtering (Kemmenoe \& Bullock, 1983; Stokroos et al., 1998) method, commonly used in SEM image analysis, involves deposition of $\mathrm{Pt}$ and $\mathrm{Au}$ ions emitted from the target onto the sample surface. Under the ion sputtering method, the shadow effect has greater influence when a metal coating is applied to dynamic structured samples, and charge-up frequently occurs. Excessive coating on the sample surface causes changes in the fine structure and even data error. This study introduces a converted sample stage to effectively remove the shadow effect, and proposes a method of high-

This work was supported by the Korea Basic Science Institute (KBSI) (Grant No. T35771).

@ This is an open-access article distributed under the terms of the Creative Commons Attribution Non-Commercial License (http://creativecommons.org/licenses/by-nc/4.0) which permits unrestricted noncommercial use, distribution, and reproduction in any medium, provided the original work is properly cited.

Copyrights @ 2015 by Korean Society of Microscopy 


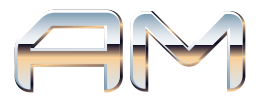

quality image analysis without any charge-up or fine structure modification.

\section{MATERIALS AND METHODS}

The sample used in the image analysis was a spherical, nonconductive $\mathrm{CO}_{2}$ absorbent ( $\sim 50 \mu$ diameter). After attaching a conductive carbon tape (TED PELLA Inc., USA; Lot. No. 16073 ) on the Al-Stub $(2.5 \mathrm{~cm})$, a $\mathrm{CO}_{2}$ absorbent was loaded on top of the carbon tape using a spatula. By blowing $\mathrm{N}_{2}$ gas, the $\mathrm{CO}_{2}$ absorbent that did not adhere to the carbon tape was removed. An ion sputter (Q150TS; Quorum Technologies, UK) was used to perform Pt ion coating under high vacuum conditions. Three kinds of sample were prepared to compare images according to the coating method. First sample was not coated, second sample was coated for 10 seconds with the stub fixed horizontally, as shown in Fig. 1A, and third sample was coated for 10 seconds in the tilt state $\left(\sim 45^{\circ}\right)$ with a rotating $\operatorname{stub}(\sim 30 \mathrm{rpm})$, as shown in Fig. 1B.

Image analysis was performed via FE-SEM (S-4700; Hitachi, Japan) in Korea Basic Science Institute (KBSI) Jeonju Center. The conditions for the image analysis were an accelerating voltage of $10 \mathrm{kV}$, ultra-high resolution lens mode, an emission current of $20 \mu \mathrm{A}$, a scan time of 40 seconds, and a working distance of $6 \sim 8 \mathrm{~mm}$.

\section{RESULTS AND DISCUSSION}

When there is no residual gas in the coater chamber, metal ions from the metal target $(\mathrm{Au}, \mathrm{Pt}$, etc.) are coated linearly on the sample surface. Fig. 2A shows ion sputtering with coating only on the linear surface (yellow layer). Due to the shadow effect, charge-up occurs in the uncoated areas (inside of red dotted line). Even when a coating is applied, the lack of a continuous coating layer results in charge-up.

As shown in Fig. 2B, residual gas such as Ar is present in the coater chamber. Metal ions from the metal target cause continuous collisions with the residual gas while moving to the surface of the sample. This collision produces a coating layer that contributes to reducing the shadow effect. When the structure of the sample surface is dynamic, charge-up occurs in the remaining uncoated areas (inside of red dotted line). The shadow effect can be eliminated by forming a thick coating layer through long time sputtering. However, the thick coating layer may cause changes to the fine structure of the sample surface, making the sample preparation inadequate for high-quality image analysis. The converted sample stage is a coating method that effectively removes the shadow effect
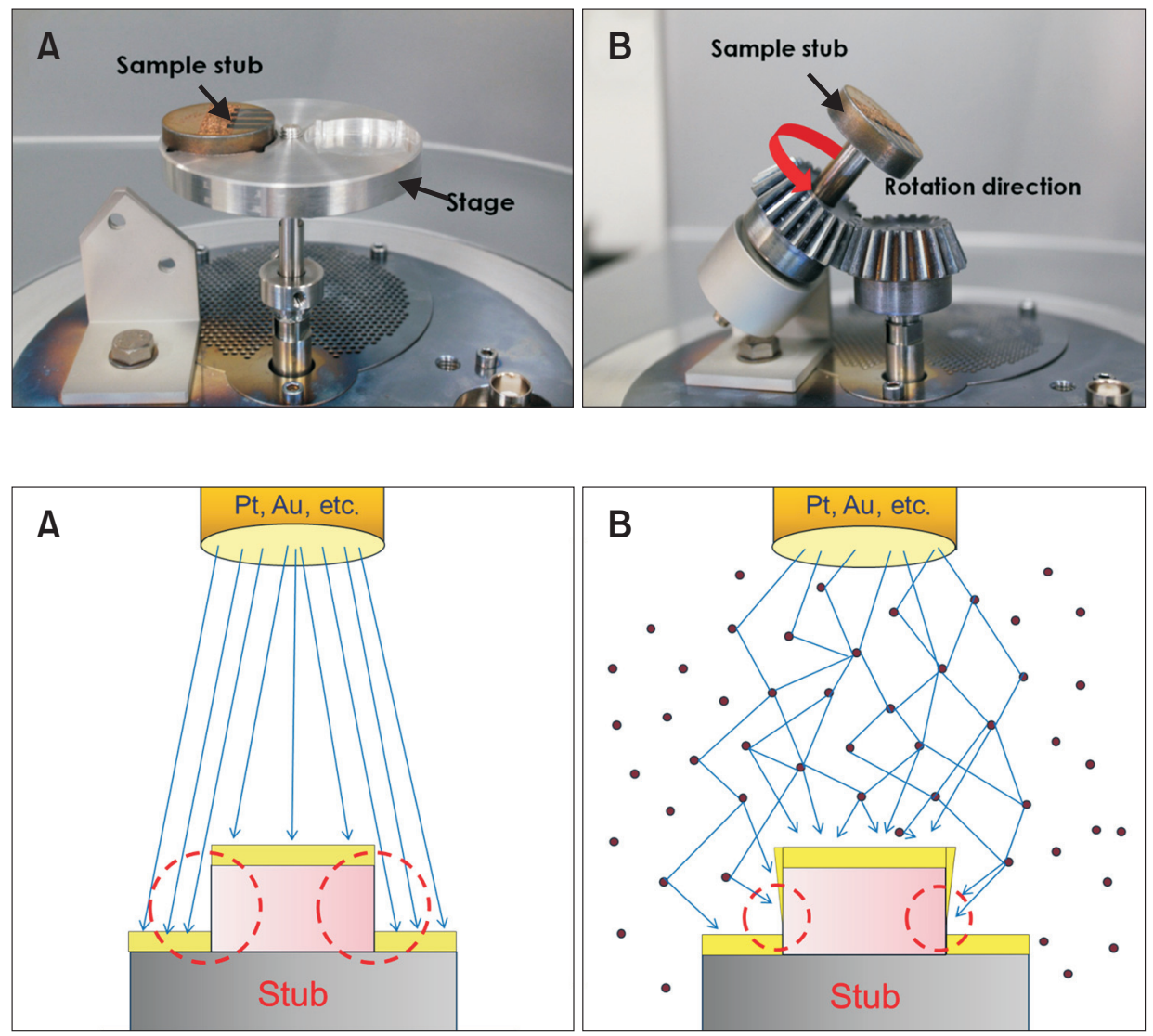

Fig. 1. Horizontal sample stage of coater (A), converted sample stage of coater; simultaneous tilt and rotation (B).
Fig. 2. Diagram of ion sputtering system; imaginary trajectory of coating particles without residual gas in chamber (A) with residual gas (B). 
while preventing modification to the fine structure of the sample surface.

When an image analysis was performed with the nonconductive, uncoated $\mathrm{CO}_{2}$ absorbent, image data acquisition was impossible due to severe charge-up, as shown in Fig. 3A. When the sample was coated using the horizontal sample stage of Fig. 1A, image data with partial charge-up were acquired, as shown in Fig. 3B.

When the sample was coated with tilt and rotation of the stub using the converted sample stage of Fig. 1B, image data without charge-up were acquired, as shown in Fig. 3C.
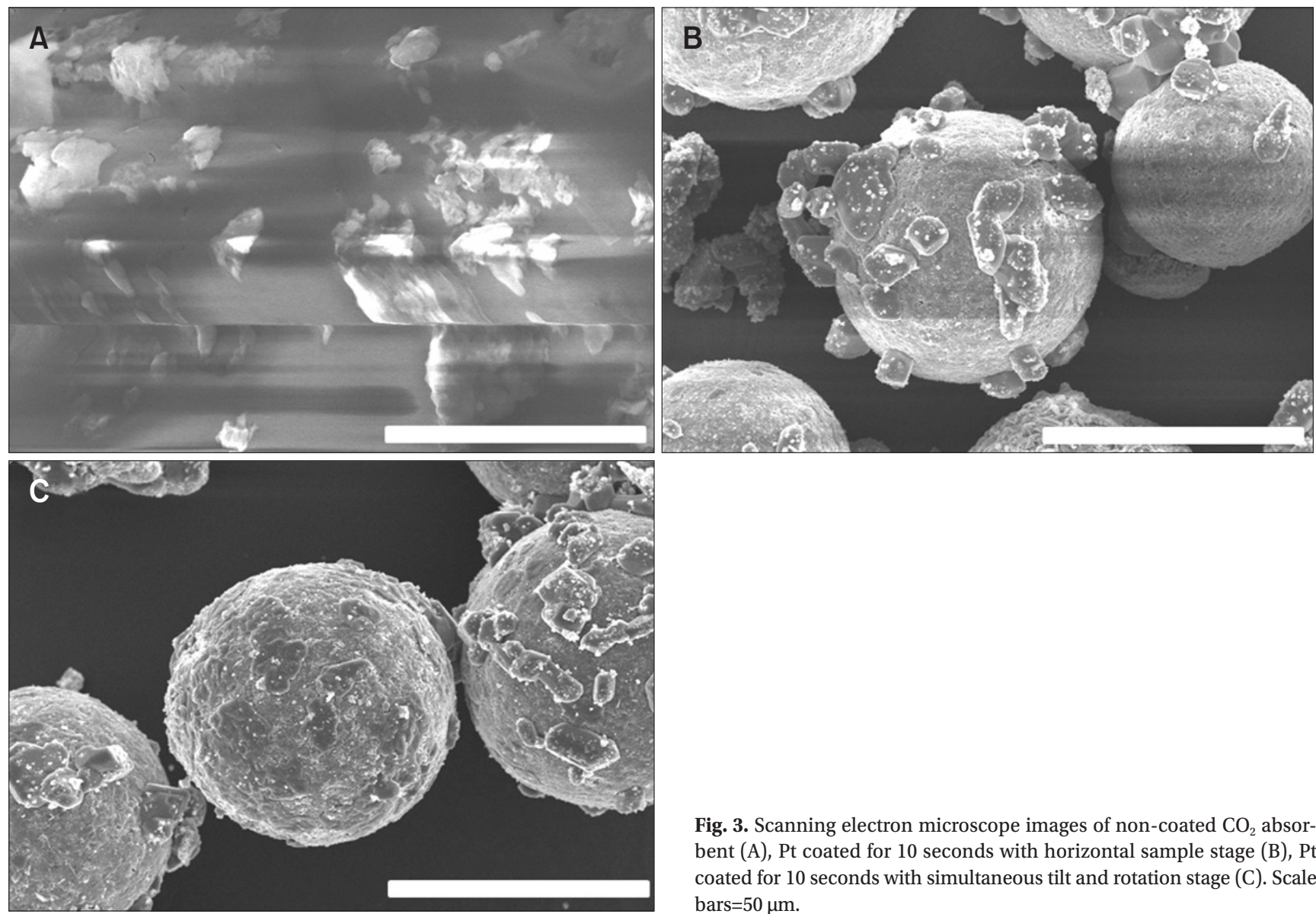

Fig. 3. Scanning electron microscope images of non-coated $\mathrm{CO}_{2}$ absorbent (A), Pt coated for 10 seconds with horizontal sample stage (B), Pt coated for 10 seconds with simultaneous tilt and rotation stage (C). Scale bars $=50 \mu \mathrm{m}$.
For the coating method of Fig. 1A, the bottom of the sphere remains uncoated due to the shadow effect, as shown in Fig. $4 \mathrm{~A}$, and this leads to charge-up. For the coating method of Fig. 1B, the tilt and rotation of the stub allows the bottom of the sphere to be fully coated, thus effectively eliminating the shadow effect and enabling images without charge-up to be acquired. The mechanism of eliminating the shadow effect is shown as Fig. 4B.

The introduction of a converted sample stage effectively eliminates the shadow effect in spherical or dynamic structured samples, and an accurate image analysis is

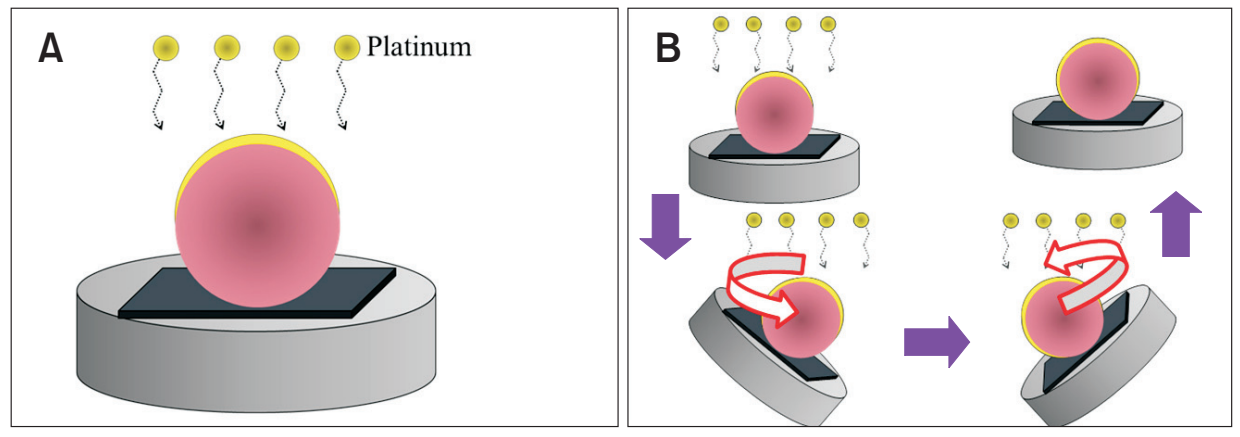


possible since no modification is made to the fine structure. The converted sample stage system can be attached by modification of the already attached sample stage.

\section{CONCLUSIONS}

The charge-up phenomenon is a frequent problem in SEM image analysis. Samples are coated to remove charge-up, but this phenomenon may still occur due to the shadow effect. An enhanced understanding of the ion sputtering mechanism, the principle behind the shadow effect, will help achieve effective coating of samples. The shadow effect can be eliminated using a converted sample stage, capable of simultaneous tilt and rotation, and this makes it possible to perform image analysis without charge-up.

\section{CONFLICT OF INTEREST}

No potential conflict of interest relevant to this article was reported.

\section{REFERENCES}

Clay C S and Peace G W (2011) lon beam sputtering: an improved method of metal coating SEM samples and shadowing CTEM samples. J. Microsc. 123, 25-34.

Egerton R F, Li P, and Malac M (2004) Radiation damage in the TEM and SEM. Micron 35, 399-409.

Hirsh P, Kassens M, Puttmann M, and Reimer L (1994) Contamination in a scanning electron microscope and the influence of specimen cooling. Scanning 16, 101-110.

Kemmenoe B H and Bullock G R (1983) Structure analysis of sputtercoated and ion-beam sputter-coated films: a comparative study. J. Microsc. 132, 153-163.

Kim K H, Akase Z, Suzuki T, and Shindo D (2010) Charging effects on $\mathrm{SEM} / \mathrm{SIM}$ contrast of metal/insulator system in various metallic coating conditions. Mater. Trans. 51, 1080-1083.
Postek M T (1996) An approach to the reduction of hydrocarbon contamination in the scanning electron microscope. Scanning $\mathbf{1 8}$, 269-274.

Shaffner T J and Van Veld R D (1990) 'Charging' effects in the scanning electron microscope. J. Phys. E: Sci. Instrum. 4, 633-637.

Stokroos I, Kalicharan D, Van Der Want J J L, and Jongebloed W L (1998) A comparative study of thin coatings of $\mathrm{Au} / \mathrm{Pd}, \mathrm{Pt}$ and $\mathrm{Cr}$ produced by magnetron sputtering for FE-SEM. J. Microsc. 189, 79-89.

Thong J T L, Lee K W, and Wong W K (2001) Reduction of charging effects using vector scanning in the scanning electron microscope. Scanning 23, 395-402.

Vladár A E and Postek M T (2005) Electron beam-induced sample contamination in the SEM. Microsc. Microanal. 11, 764-765. 